\title{
Chance-Constrained Peer-to-Peer Joint Energy and Reserve Market Considering Renewable Generation Uncertainty
}

Guo, Zhenwei; Pinson, Pierre; Chen, Shibo; Yang, Qinmin; Yang, Zaiyue

Published in:

IEEE Transactions on Smart Grid

Link to article, DOI:

10.1109/TSG.2020.3019603

Publication date:

2021

Document Version

Peer reviewed version

Link back to DTU Orbit

Citation (APA):

Guo, Z., Pinson, P., Chen, S., Yang, Q., \& Yang, Z. (2021). Chance-Constrained Peer-to-Peer Joint Energy and Reserve Market Considering Renewable Generation Uncertainty. IEEE Transactions on Smart Grid, 12(1), 798 809. https://doi.org/10.1109/TSG.2020.3019603

\section{General rights}

Copyright and moral rights for the publications made accessible in the public portal are retained by the authors and/or other copyright owners and it is a condition of accessing publications that users recognise and abide by the legal requirements associated with these rights.

- Users may download and print one copy of any publication from the public portal for the purpose of private study or research.

- You may not further distribute the material or use it for any profit-making activity or commercial gain

- You may freely distribute the URL identifying the publication in the public portal 


\title{
Chance-Constrained Peer-to-Peer Joint Energy and Reserve Market Considering Renewable Generation Uncertainty
}

\author{
Zhenwei Guo, Student Member, IEEE, Pierre Pinson, Fellow, IEEE, Shibo Chen, Member, IEEE, \\ Qinmin Yang, Member, IEEE, and Zaiyue Yang, Member, IEEE
}

\begin{abstract}
Due to the fast development of distributed energy resources and demand-side response management, agents in electricity markets are becoming more proactive, which boosts the development of peer-to-peer (P2P) market mechanisms. However, to our knowledge, none of the existing works considers clearing both energy and reserve via a P2P market mechanism in order to compensate for the uncertainty originating from renewable generation and allocate the reserve cost induced by uncertainty fairly. In this paper, a novel P2P joint energy and reserve market is proposed, where each agent can negotiate with neighboring agents to determine the quantities and prices of traded energy and reserve. We model the renewable generation uncertainty by versatile distribution and determine the required reserve based on a chance-constrained optimization approach. Then, a fully decentralized P2P market based on consensus alternating direction method of multipliers (ADMM) theory is proposed. In addition, to further lower the social cost, we exploit the correlation and complementarity among uncertainties and design a renewable community-based market, where all renewable agents share uncertainty information to community manager for calculating total required reserve. Finally, simulation results show the convergence performance, fairness and scalability of our market mechanism.
\end{abstract}

Index Terms-Peer-to-peer market, joint energy and reserve market, consensus ADMM, chance-constrained, uncertainty correlation, versatile distribution, Gaussian mixture model

\section{NOMENCLATURE}

\section{Functions}

$\tilde{C}(\cdot) \quad$ Bilateral trading cost.

$C(\cdot) \quad$ Production cost or utility function.

$f, F, F^{-1}$ PDF, CDF and inverse function of CDF.

$N(\cdot) \quad$ Multivariate Gaussian distribution function.

Numbers and Indexes

Manuscript received February 29, 2020; revised July 7 and August 19, 2020; accepted August 22, 2020. This work was supported in part by National Natural Science Foundation of China (61873118, 61903179, 61673347, 61751205), in part by Shenzhen Committee on Science and Innovations under Grant GJHZ20180411143603361, in part by Department of Science and Technology of Guangdong Province under Grant 2018A050506003, in part by Key-Area Research and Development Program of Guangdong Province (No. 2018B010107002). Paper no. TSG-00297-2020. (Corresponding author: Z. Yang.)

Z. Guo and Q. Yang are with the State Key Laboratory of Industrial Control Technology, Zhejiang University, Hangzhou 310027, China (e-mail: zhenweiguo0724@163.com, qmyang@zju.edu.cn).

S. Chen and Z. Yang are with Department of Mechanical and Energy Engineering, Southern University of Science and Technology, Shenzhen 518055, China (e-mail: chensb@ sustech.edu.cn, yangzy3@ sustc.edu.cn)

P. Pinson is with the Center for Electric Power and Energy, Danmarks Tekniske Universitet, Denmark, e-mail: ppin@dtu.dk.

$\begin{array}{ll}e, r & \text { Indices for energy and reserve. } \\ i, j & \text { Indices for buses. } \\ k & \text { Index for iterations. } \\ L & \text { Cardinal number of components. } \\ l & \text { Index for Gaussian components. } \\ n, m & \text { Indices for agents. } \\ s, t & \text { Primal and dual residuals. }\end{array}$

\section{Parameters}

$\alpha, \beta, \gamma$ Shape parameters for versatiole distributon.

$\chi \quad$ Stopping criterian.

$\epsilon \quad$ Acceptable probability.

$\kappa^{i n c r}, \kappa^{\text {decr }}$ Increasing and decreasing factors for adaptive penalty factors.

$\mathbb{R} \quad$ Total required reserve for the renewable community.

$\rho, \tau, \phi$ Penalty factors.

$\tilde{R} \quad$ Actual uncertainty.

$\underline{E}, \bar{E} \quad$ Boundaries of power.

$\underline{R}, \bar{R} \quad$ Boundaries of reserve.

$\varpi, \mu, \Sigma$ Weight coefficient, mean vector and correlation matrix.

$a, b \quad$ Coefficients of the quadractic fucntions.

$c \quad$ Bilateral trading coefficient.

$E^{f} \quad$ Forcast power generation of renewable agent.

$F \quad$ Fairness of reserve payment dispatch.

$h \quad$ Coefficients of the fourth-order polynomials.

$p \quad$ Payment.

poly A fourth-order polynomial.

$\operatorname{poly}_{\Sigma}$ The summation of multiple fourth-order polynomials.

$u \quad$ Normalized uncertainty.

$Y \quad$ Whole uncertainty random variable that is the linear summation of $\tilde{\mathbf{R}}$.

$y \quad$ Value of $Y$.

Sets and Vectors

$\Gamma \quad$ Adjustable parameter set of a Gaussian mixture model (GMM).

$\tilde{\mathbf{R}}$ Random vector of multiple uncertainty distributions.

E,R Vector of whole transactions.

$\mathbf{P} \quad$ Vector of whole power flows.

$\mathcal{L} \quad$ Set of lines.

$\mathcal{N} \quad$ Set of buses.

$\Omega \quad$ Set of agents.

$\omega \quad$ Set of neighboring agents.

$\Omega_{g}, \Omega_{r}, \Omega_{u}$ Set of conventional generators, renewable generators and users.

Variables 
$\lambda, \nu \quad$ Energy and reserve prices.

$\theta \quad$ Voltage angle.

$v \quad$ Dual variable corresponding to power balance.

$\varrho \quad$ Dual variable corresponding to constraint (1).

$E \quad$ Power injection or traded quantity.

$P \quad$ Power flow.

$R \quad$ Reserve injection or traded quantity.

\section{INTRODUCTION}

The ever-increasing distributed energy resources and energy system management are changing the approach of power system operation. Individual participants are becoming more proactive in the market, who prefer determining the trading outcomes by themselves. Therefore, electricity markets are expected to evolve towards more decentralized mechanisms. However, current electricity markets still perform resource allocation and pricing based on the conventional hierarchical and top-down approach [1], which makes prosumers behave as passive receivers. Recently, a novel design of energy trading and electricity market mechanisms has emerged: these socalled peer-to-peer (P2P) trading mechanisms rely on multibilateral trades between each pair of participants [2]-[15]. Employing a P2P trading mechanism can yield plenty of advantages, e.g., empowerment of participants, increasing the reliability of power system and protection of privacy [11]. Existing works about P2P trading and markets mainly focus on the transaction of electrical energy, with the consideration of: distribution networks [3], [4], [9], [10], reallocation of the costs [9], product differences [4], [10], [15], dispatch fairness [14], blockchain [2], [3], electric vehicles [2], [5], microgrid [6], [7] and communication burden [12], [13]. Different decentralized methods are employed to realize market mechanism, for instance, primal-dual gradient [10], relaxed consensus+innovation [12], [15], standard ADMM [4], [14], bilateral contract [8], game theory [6], [7], and consensus ADMM [9], [13]. However, to the best of our knowledge, none of the existing works considers trading the reserve also via a $\mathrm{P} 2 \mathrm{P}$ mechanism.

With the rapid penetration of renewable generation and autonomous demand adjustment by smart appliances, the random fluctuation and uncertainty of power systems at both generation and demand sides have experienced a significant increase in recent years, which severely threaten the stability, efficiency, and reliability of power systems. In order to compensate for the fluctuation and uncertainty, a large quantity of reserve is in an urgent need in the era of smart grid. Indeed, reserve is regarded as an important resource to guarantee the proper operation of power systems besides energy [16]-[18].

Nowadays, the reserve is usually procured by the system operator (SO) to make sure the security of power systems. This framework is workable for now since the required reserve is not much, and the uncertainty is not deliberately brought by generators and consumers. However, with the massive increase of distributed renewable energy, the demanded quantity for reserve becomes larger and lager. Meanwhile, the renewable agents can forecast how much uncertainty they will bring, and they are also responsible for compensating the uncertainty. It is unreasonable and uneconomical to still require
SO ensure sufficient reserve. We believe it is sound to also clear the reserve in a distributed/decentralized market way, which can allocate the cost induced by providing reserve fairly and increase the quality of renewable generation. The work [19] also considered that there should be some mechanisms to enforce accountability for the additional costs induced by power fluctuations. The authors proposed an attribution mechanism for fairly redistributing related power regulation costs (reserve costs). From an economic point of view, the renewable agents instead of the SO should pay for the reserve payments. While from the system operation perspective, it still be the $\mathrm{SO}$ who decides how to use and dispatch the reserve. Thus, the P2P market mechanism just reallocates the reserve cost in a fair way, and does not break the existing system operation mechanism.

Only few works about P2P markets consider the trading of reserve. For example, [20] proposes a P2P coordinated market for energy trade and ancillary services, but the reserve is still cleared in a centralized way. Another work [21] considers a three-stage community-based P2P market with reserve activation, but the reserve is provided and bidden individually into reserve market instead of trading between peers.

Summing up the above, the challenging problem we are trying to solve is how to establish a proper electricity market that can clear both energy and reserves within a P2P mechanism to cover uncertainties and allocate the corresponding reserve costs in a fair way. To this end, we propose a novel P2P joint energy and reserve market, which can clear day-ahead energy and reserve dispatches simultaneously. The renewable generation uncertainty is modeled via a versatile distribution [22] and quantify the needed reserve based on a chance-constrained optimization approach. Then, a fully decentralized negotiation mechanism based on consensus ADMM is proposed. In addition, to further lower the social cost, we exploit the correlation and complementarity among renewable generation uncertainties, and design a modified renewable communitybased P2P joint market, where all renewable agents share their uncertainty information to a community manager to calculate the total required reserve. The uncertainty of all renewable agents is characterized by a Gaussian mixture model (GMM). Finally, in the simulations, we show the fairness by comparing our markets with the pool-based market, validate the convergence performance by running a large case with real wind power generation data, and demonstrate the scalability by increasing the number of agents. The contributions mainly lie as follows.

- We propose a novel P2P joint energy and reserve market, where all agents can freely negotiate with neighboring agents over the quantities and prices of energy and reserve. This fully decentralized market enables energy clearing and uncertainty covering simultaneously. Besides, the reserve cost induced by the uncertainty can be allocated to each renewable agent in a more fair way.

- By employing the chance-constrained optimization approach and the versatile distribution, we accurately model the renewable generation uncertainty and quantify the required reserve.

- In order to utilize the correlation and complementarity 
among uncertainties to reduce the social cost, we propose a modified renewable community-based $\mathrm{P} 2 \mathrm{P}$ joint market, where all renewable agents collaborate and share uncertainty information to the community manager.

The rest of the paper is organized as follows: Section II presents the chance-constrained social cost minimization problem. Section III proposes the full P2P joint market, followed by the renewable community-based P2P joint market in Section IV. Numerical results and comparisons are presented in Section V. Finally, conclusions, limitations and future perspectives are drawn in Section VI.

\section{PRoblem Formulation}

We consider a market with a set $\Omega$ of agents who are defined as either energy/reserve producers or consumers. Similar to energy consumers who need the energy to satisfy their demand, the reserve consumers also need the reserve to compensate for their uncertainty. We assume that the uncertainty only comes from renewable agents and only deficiency case is considered since the surplus generation can always be cut down. All agents are supposed to be rational and truthful as in [23], which means they always make strategic decisions to maximize individual benefits. The market mechanism proposed in the following is for a day-ahead single slot market. Without loss of generality, we assume that the length of time slot is 1 hour, which means the values of energy and power are same.

\section{A. Peer-to-Peer Trading}

A P2P mechanism for electricity markets is much more decentralized compared with existing centralized markets. For the centralized market, all agents have to provide their whole information, e.g., cost or utility function, power limits and uncertainty to the SO, who will centrally determine the dispatches of energy and reserve. However, in P2P market, all agents can freely negotiate the prices and quantities of multi-bilateral trades between each other. P2P mechanism involves players directly while respecting data privacy and being resistant to the failure or quit of any player.

To model the trading process, the net power injection $E_{n}$ of each agent $n \in \Omega$ is split into a sum of bilaterally traded quantities with a set of neighboring agents $m \in \omega_{n}$ as

$$
E_{n}=\sum_{m \in \omega_{n}} E_{n m}, \quad \forall n \in \Omega
$$

A positive value of $E_{n m}$ corresponds to a sale/production and a negative value to a purchase/consumption. The set $\left\{E_{n m} \mid n \in \Omega, m \in \omega_{n}\right\}$ is the set of decision variables. To lighten notations, $\mathbf{E}_{n}=\left\{E_{n 1}, \ldots, E_{n m}\right\}$ is used to represent the whole set of transactions of agent $n$. The power set-points of an agent $n$ is constrained by the boundaries $\underline{E_{n}}$ and $\overline{E_{n}}$,

$$
\underline{E_{n}} \leq E_{n} \leq \overline{E_{n}}, \quad \forall n \in \Omega
$$

Each agent is restrained to either producer or consumer $\left(\underline{E_{n}} \overline{E_{n}} \geq 0\right)$. Hence, the decision variables are constrained to be positive $\left(E_{n m} \geq 0\right)$ if it is a producer or negative $\left(E_{n m} \leq 0\right)$ if it is a consumer as

$$
\begin{cases}E_{n m} \geq 0, & \forall(n, m) \in\left(\Omega_{g} \cup \Omega_{r}, \omega_{n}\right) \\ E_{n m} \leq 0, & \forall(n, m) \in\left(\Omega_{u}, \omega_{n}\right)\end{cases}
$$

where $\Omega_{g}, \Omega_{r}$, and $\Omega_{u}$ are the sets of conventional generators, renewable generators and users, respectively. We assume the producer generation cost or consumer utility $C_{n}^{e}\left(E_{n}\right)$ (positive for generators while negative for users) are strictly convex functions.

Similarly, the reserve injection $R_{n}$ of each agent is split as

$$
R_{n}=\sum_{m \in \omega_{n}} R_{n m}, \quad \forall n \in \Omega
$$

The set $\left\{R_{n m} \mid n \in \Omega, m \in \omega\right\}$ is the set of reserve decision variables, $\mathbf{R}_{n}=\left\{R_{n 1}, \ldots, R_{n m}\right\}$ is used to represent the whole reserve transactions. The reserve level of an agent $n$ is constrained by the boundaries $\underline{R_{n}}$ and $\overline{R_{n}}$,

$$
\underline{R_{n}} \leq R_{n} \leq \overline{R_{n}}, \quad \forall n \in \Omega
$$

Each agent is restrained to either reserve provider or consumer $\left(R_{n} \overline{R_{n}} \geq 0\right)$. Hence, the decision variables of agent are constrained to be positive $\left(R_{n m} \geq 0\right)$ if it is a reserve provider or negative $\left(R_{n m} \leq 0\right)$ if it is a reserve consumer as

$$
\begin{cases}R_{n m} \geq 0, & \forall(n, m) \in\left(\Omega_{g} \cup \Omega_{u}, \omega_{n}\right) \\ R_{n m} \leq 0, & \forall(n, m) \in\left(\Omega_{r}, \omega_{n}\right)\end{cases}
$$

We also assume the reserve production cost or consumer utility function $C_{n}^{r}\left(R_{n}\right)$ (negative for renewable generators while positive for others) as strictly convex functions. Here, we believe the concept "reserve utility" is meaningful and helpful. The SO can encourage renewable agents to improve the quality of generation by punishing the shortage or designing incentive mechanism. If the renewable agents can generate power accurately to ensure the safety of power systems, they can avoid penalty or receive subsidy, which can be regarded as the "reserve utility". Early works [24], [25] also proposed the penalty cost for wind power plant failing to provide the scheduled power. Besides, by proposing this "reserve utility", each renewable generator has the motivation to improve the generation quality, which will result in stability improvement of power systems.

Adequate amount of reserve is needed even at the worst case, i.e.,

$$
\underline{E_{n}} \leq E_{n}+R_{n} \leq \overline{E_{n}}, \quad \forall n \in \Omega
$$

Finally, the market equilibrium between energy/reserve production and consumption is represented by balance constraints, and in P2P market, they can be replaced by a set of reciprocity constraints defined as

$$
\begin{cases}E_{n m}+E_{m n}=0, & \forall(n, m) \in\left(\Omega, \omega_{n}\right) \\ R_{n m}+R_{m n}=0, & \forall(n, m) \in\left(\Omega, \omega_{n}\right)\end{cases}
$$




\section{B. Power Network Model}

Let the power network consist of a set $\mathcal{N}$ of buses and a set $\mathcal{L}$ of lines. We consider the DC power flow model to characterize the line limits and the generation-load balance, which is widely used in market studies [26], [27]. Let $P_{i j}$ be the real power flow in the line connecting bus $i$ and $j, Y_{i j}$ is the susceptance of the line, $\theta_{i}$ is the voltage angle at bus $i$, and $C_{i j}$ is the line thermal limit. Without the loss of generality, let bus 1 be the slack bus, i.e., $\theta_{1}=0$. Then, the network constraint and power balance can be represented as follows,

$$
\begin{aligned}
-C_{i j} \leq P_{i j}=Y_{i j}\left(\theta_{j}-\theta_{i}\right) \leq C_{i j}, & \forall(i, j) & \in \mathcal{L} \\
\sum_{n \in \mathcal{N}_{i}} E_{n} & =\sum_{(i, j) \in \mathcal{L}} P_{i j}, & \forall i \in \mathcal{N}
\end{aligned}
$$

In transmission networks, the real power flows $P_{i j}$ are proportional to the difference of voltage angles between the two ends of the line. To avoid any damage to transmission lines, the power flows are bounded by thermal capacity $C_{i j}$ related to the heat they can dissipate. Moreover, the power balance (9b) must be kept at each bus of the grid between line flows and power injections of agents connected to it. $\mathbf{P}=\left\{P_{i j},(i, j) \in \mathcal{L}\right\}$ and $\boldsymbol{\theta}=\left\{\theta_{i}, i \in \mathcal{N}\right\}$ are used to represent all the power flows in lines and voltage angles of buses.

\section{Product differentiation}

The product differentiation is also considered in our joint P2P market, inspired by [15], the bilateral trading costs are calculated as linear functions of the quality traded with each neighboring agents,

$$
\tilde{C}_{n}^{e}\left(\mathbf{E}_{n}\right)=\sum_{m \in \omega_{n}} c_{n m}^{e} E_{n m}, \quad \tilde{C}_{n}^{r}\left(\mathbf{R}_{n}\right)=\sum_{m \in \omega_{n}} c_{n m}^{r} R_{n m}
$$

The bilateral trading coefficients $\left\{c_{n m}^{e}, c_{n m}^{r}\right\}$ are expressed for the purpose of product differentiation, which is affected by emissions, transport distance, size of prosumers, etc.

\section{Chance-constrained Social Cost Minimization Problem}

From above, the P2P joint market has the objective to minimize the social cost of all agents under the constraints. The problem is formulated as

$$
\begin{aligned}
\min & \sum_{n \in \Omega}\left(C_{n}^{e}\left(E_{n}\right)+\tilde{C}_{n}^{e}\left(\mathbf{E}_{n}\right)+C_{n}^{r}\left(R_{n}\right)+\tilde{C}_{n}^{r}\left(\mathbf{R}_{n}\right)\right) \\
\text { s.t. } & (1)-(9) \\
& \mathbb{P}\left\{\tilde{R}_{n} \geq R_{n}\right\} \geq 1-\epsilon_{n}, \quad \forall n \in \Omega
\end{aligned}
$$

For renewable agents, the generation power is fixed to the forecast value. Once the forecast power is given, the corresponding forecast uncertainty distribution is obtained, based on which, the required reserve for each renewable agent is determined using the chance-constrained approach. (11b) implies that the probability of the actual uncertainty $\tilde{R}_{n}$ within the scheduled reserve $R_{n}$, is not less than one by an acceptable probability $\epsilon_{n}$ decided by each agent individually.

Since the social cost minimization problem is a convex optimization problem, it has unique optimum, which can be

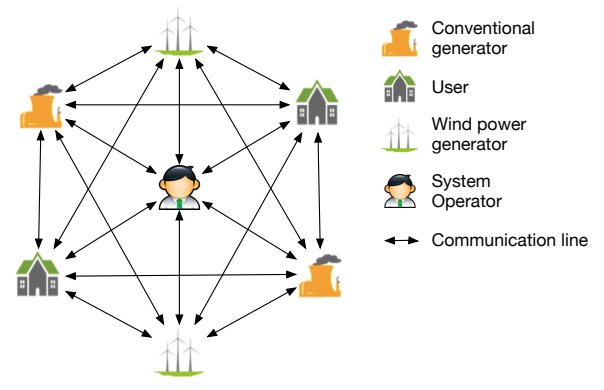

Fig. 1: Full P2P joint market with SO

obtained by a wealth of centralized methods. However, it requires the exposure of all the information of agents, thus cannot preserve privacy. To this end, a $\mathrm{P} 2 \mathrm{P}$ mechanism is more desirable. Next, we are going to design a fully decentralized P2P joint market that can achieve the optimal dispatches of above social cost minimization problem (11).

\section{Full P2P Joint Market}

In the full P2P joint market, each agent can negotiate with neighbors to trade energy and reserve, and each renewable agent can determine the needed reserve individually without sharing private uncertainty information. To this end, we need to convert the probability form of the chance constraint (11b) into a deterministic form compatible with (11). Besides, there is a SO who behaves like a single agent in the market, to help complete the power flows and voltage angles calculation. Then, a decentralized negotiation mechanism based on the consensus ADMM is designed. The market structure of our full P2P joint market is shown in Fig. 1.

\section{A. Modeling Uncertainty and Determining Reserve}

The first problem is how to well represent the renewable generation uncertainty, which is usually assumed as a Gaussian distribution [28]. However, many studies have demonstrated that Gaussian distribution cannot always model uncertainty accurately. Hence, a prior work [22] proposed a "versatile distribution", whose probability density function (PDF), cumulative distribution function (CDF) and inverse function of $\mathrm{CDF}$ are as

$$
\begin{gathered}
f(x \mid \alpha, \beta, \gamma)=\frac{\alpha \beta e^{-\alpha(x-\gamma)}}{\left(1+e^{-\alpha(x-\gamma)}\right)^{\beta+1}} \\
F(x \mid \alpha, \beta, \gamma)=\left(1+e^{-\alpha(x-\gamma)}\right)^{-\beta} \\
F^{-1}(c \mid \alpha, \beta, \gamma)=\gamma-\frac{1}{\alpha} \ln \left(c^{-1 / \beta}-1\right)
\end{gathered}
$$

where $\alpha, \beta$, and $\gamma$ are the shape parameters and $c$ is the confidence level. The shape parameters can be determined by curve fitting based on nonlinear least squares estimation. A pictorial example is shown in Fig. 2. Seen from the figures, the versatile distribution can fit the actual $\mathrm{CDF}$ and inverse CDF better than the Gaussian distribution.

Two reasons for choosing versatile distribution over Gaussian distribution are: 1) The versatile distribution can well represent renewable generation uncertainty CDF and inverse 


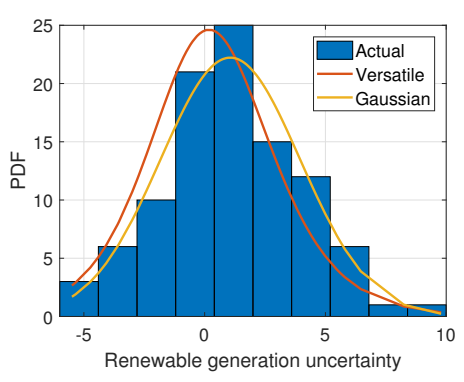

(a)

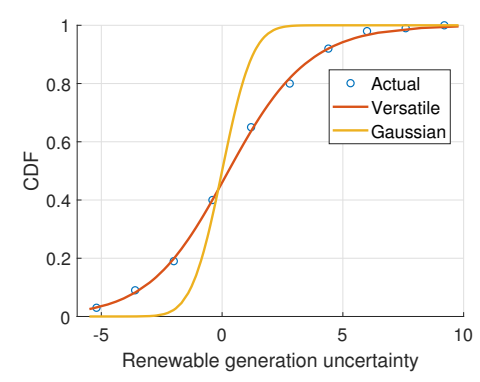

(b)

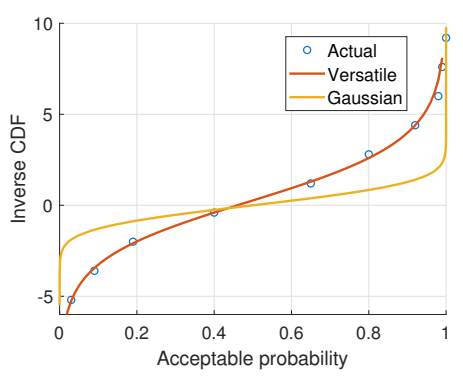

(c)

Fig. 2: A pictorial example of the versatile distribution

CDF. 2) The CDF and its inverse function have analytical forms. Thus, the required reserve can be calculated as follows:

$$
\begin{aligned}
& \mathbb{P}\left\{\tilde{R}_{n} \geq R_{n}\right\}=1-F\left(R_{n}\right) \geq 1-\epsilon_{n} \\
& \Rightarrow R_{n} \leq F^{-1}\left(\epsilon_{n}\right)=\gamma-\frac{1}{\alpha} \ln \left(\epsilon_{n}^{-1 / \beta}-1\right)
\end{aligned}
$$

Thus we can fix the boundaries $\underline{R_{n}}=\overline{R_{n}}=\gamma-\frac{1}{\alpha} \ln \left(\epsilon_{n}^{-1 / \beta}-1\right)$ for each renewable agent $n \in \overline{\Omega_{r}}$.

Remark 1. Although the versatile distribution method is proposed to model the uncertainty from wind power [22], many early works have demonstrated that the uncertainty from wind and solar power has similar profiles of PDF and CDF [29]-[31]. Thus this method can be adopted to model different types of renewable generation uncertainty.

\section{B. Decentralized Negotiation Mechanism}

ADMM is an algorithm that blends the decomposability of dual ascent with the superior convergence properties of the method of multipliers, but there is a central coordinator for updating the dual variable. Thus, in order to deploy ADMM into our P2P joint market, we adopt the consensus ADMM method [32], which incorporates the decentralized property of consensus-based approach. As developed in Appendix A, a decentralized negotiation mechanism based on the consensus ADMM is used to solve (11).

In addition, the SO will be added as a single agent to complete the power flows and voltage angles calculation. The network constraints (9a)-(9b) can be converted into an optimization problem as

$$
\left\{\mathbf{P}^{k+1}, \boldsymbol{\theta}^{k+1}\right\}=\underset{\{\mathbf{P}, \boldsymbol{\theta}\}}{\operatorname{argmin}} \sum_{i \in \mathcal{N}}\left\|\sum_{n \in \mathcal{N}_{i}} E_{n}^{k}-\sum_{(i, j) \in \mathcal{L}} P_{i j}\right\|_{2}^{2}
$$

$$
\text { s.t. } \quad-C_{i j} \leq P_{i j}=Y_{i j}\left(\theta_{j}-\theta_{i}\right) \leq C_{i j} \quad(i, j) \in \mathcal{L}
$$

At each step of the negotiation mechanism, the SO first collects the total power injection $E_{n}^{k}, n \in \Omega$ from all agents located at different buses, then determines the power flow $\mathbf{P}^{k+1}$ and voltage angles $\boldsymbol{\theta}^{k+1}$, and finally returns them to all agents. Then for each agent $n$ in bus $i$, the local optimization problem at a given iteration $k$ is

$$
\left\{\mathbf{E}_{n}^{k+1}, \mathbf{R}_{n}^{k+1}\right\}=
$$

$\operatorname{argmin} C_{n}^{e}\left(E_{n}\right)+\tilde{C}_{n}^{e}\left(\mathbf{E}_{n}\right)+C_{n}^{r}\left(R_{n}\right)+\tilde{C}_{n}^{r}\left(\mathbf{R}_{n}\right)+$ $\left\{\mathbf{E}_{n}, \mathbf{R}_{n}\right\}$

$$
\begin{aligned}
& \sum_{m \in \omega_{n}}\left[\lambda_{n m}^{k}\left(\frac{E_{n m}^{k}-E_{m n}^{k}}{2}-E_{n m}\right)+\nu_{n m}^{k}\left(\frac{R_{n m}^{k}-R_{m n}^{k}}{2}-R_{n m}\right)\right. \\
& +\frac{\rho}{2}\left(\frac{E_{n m}^{k}-E_{m n}^{k}}{2}-E_{n m}\right)^{2}+\frac{\tau}{2}\left(\frac{R_{n m}^{k}-R_{m n}^{k}}{2}-R_{n m}\right)_{(17 \mathrm{a})}^{2} \\
& +v_{i}^{k}\left(E_{n}+E_{-n}^{k}-\sum_{(i, j) \in \mathcal{L}} P_{i j}^{k+1}\right)+\frac{\phi}{2}\left(E_{n}+E_{-n}^{k}-\sum_{(i, j) \in \mathcal{L}} P_{i j}^{k+1}\right)^{2}
\end{aligned}
$$

$$
\text { s.t. } \quad(1)-(7)
$$

where $E_{-n}^{k}$ denotes the summation of power injection of all agents other than $n$ at bus $i ; \rho, \tau$ and $\phi$ are positive penalty factors; $\lambda_{n m}$ and $\nu_{n m}$ are the dual variables of the reciprocity constraints (8), also define the prices for the traded energy power $E_{n m}$ and reserve $R_{n m} ; v_{i}$ is the dual variable corresponding to constraint (9b). $\boldsymbol{\lambda}_{n}=\left\{\lambda_{n 1}, \ldots, \lambda_{n m}\right\}$ and $\boldsymbol{\nu}_{n}=\left\{\nu_{n 1}, \ldots, \nu_{n m}\right\}$ are used to represent the whole energy and reserve prices between neighboring agents of agent $n$; $\boldsymbol{v}=\left\{v_{i}, i \in \mathcal{N}\right\}$ is used to represent the whole dual variables for constraint (9b). Then the energy price $\lambda_{n m}^{k+1}$, reserve price $\nu_{n m}^{k+1}$, and dual variable $v_{i}^{k+1}$ will be updated as

$$
\begin{aligned}
\lambda_{n m}^{k+1} & =\left[\lambda_{n m}^{k}-\rho\left(E_{n m}^{k+1}+E_{m n}^{k+1}\right) / 2\right]^{+} \\
\nu_{n m}^{k+1} & =\left[\nu_{n m}^{k}-\tau\left(R_{n m}^{k+1}+R_{m n}^{k+1}\right) / 2\right]^{+} \\
v_{i}^{k+1} & =\left[v_{i}^{k}+\phi\left(\sum_{n \in \mathcal{N}_{i}} E_{n}^{k+1}-\sum_{(i, j) \in \mathcal{L}} P_{i j}^{k+1}\right)\right]^{+}
\end{aligned}
$$

where $[.]^{+}$denotes the $\max (., 0)$. Since the $C_{n}^{e}\left(E_{n}\right)$ and $C_{n}^{r}\left(R_{n}\right)$ are closed, proper and convex, $\tilde{C}_{n}^{e}\left(\mathbf{E}_{n}\right)$ and $\tilde{C}_{n}^{r}\left(\mathbf{R}_{n}\right)$ are linear, and the objective function of SO is also convex, it is sufficient to ensure convergence to the global optimal point of the ADMM method [32]. The market converges as long as the total local residuals and the objective function of SO (16a) fall below global stopping criteria,

$$
\begin{aligned}
& S E^{k+1} \triangleq \sum_{n \in \Omega} s_{n, e}^{k+1} \leq \chi_{e}^{s}, S R^{k+1} \triangleq \sum_{n \in \Omega} s_{n, r}^{k+1} \leq \chi_{r}^{s} \\
& T E^{k+1} \triangleq \sum_{n \in \Omega} t_{n, e}^{k+1} \leq \chi_{e}^{t}, T R^{k+1} \triangleq \sum_{n \in \Omega} t_{n, r}^{k+1} \leq \chi_{r}^{t}
\end{aligned}
$$




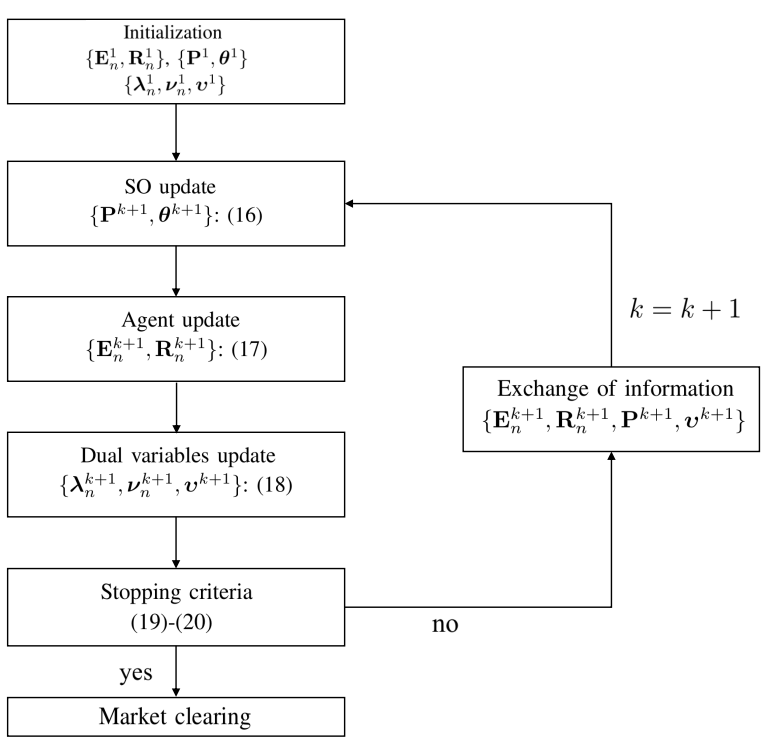

Fig. 3: Flowchart of the decentralized negotiation mechanism for full P2P joint market

$$
S O^{k+1} \triangleq \sum_{i \in \mathcal{N}}\left\|\sum_{n \in \mathcal{N}_{i}} E_{n}^{k+1}-\sum_{(i, j) \in \mathcal{L}} P_{i j}^{k+1}\right\|_{2}^{2} \leq \chi_{s o}
$$

where the primal and dual local residuals are defined as

$$
\begin{aligned}
& s_{n, e}^{k+1} \triangleq \sum_{m \in \omega_{n}}\left(E_{n m}^{k+1}+E_{m n}^{k+1}\right)^{2}, s_{n, r}^{k+1} \triangleq \sum_{m \in \omega_{n}}\left(R_{n m}^{k+1}+R_{m n}^{k+1}\right)^{2} \\
& t_{n, e}^{k+1} \triangleq \sum_{m \in \omega_{n}}\left(E_{n m}^{k+1}-E_{n m}^{k}\right)^{2}, t_{n, r}^{k+1} \triangleq \sum_{m \in \omega_{n}}\left(R_{n m}^{k+1}-R_{n m}^{k}\right)^{2}
\end{aligned}
$$

The decentralized negotiation mechanism is detailed in Fig. 3.

Remark 2. This market mechanism can be readily extended to multiple time slots with time-coupling constraints, like robust ramping rate limits and total demand requirement. These constraints are not coupled among agents, which means they can be decoupled and included into the local optimization problem (17) without breaking the convexity of the local problem. Thus, our single-time slot P2P joint market mechanism is still applicable with time-coupling constraints.

\section{Algorithm Acceleration}

The biggest problem for deploying and operating P2P mechanisms into the real electricity market is the heavy burden of communication between agents. In theory, ADMM converges for any constant penalty factor. In practice, however, the performance and reliability of ADMM depend heavily on the choice of penalty factor, and it can be improved by adaptive penalty selection methods.

$$
\rho^{k+1}:= \begin{cases}\kappa^{i n c r} \rho^{k} & \text { if } S E^{k}>10 * T E^{k} \\ \rho^{k} / \kappa^{\text {decr }} & \text { if } T E^{k}>10 * S E^{k} \\ \rho^{k} & \text { otherwise }\end{cases}
$$

$$
\tau^{k+1}:= \begin{cases}\kappa^{i n c r} \tau^{k} & \text { if } S R^{k}>10 * T R^{k} \\ \tau^{k} / \kappa^{\text {decr }} & \text { if } T R^{k}>10 * S R^{k} \\ \tau^{k} & \text { otherwise }\end{cases}
$$

The iterations are accelerated by varying the penalty factors $\rho^{k}$ and $\tau^{k}$ according to (21)-(22). $\kappa^{\text {incr }}$ and $\kappa^{\text {decr }}$ are increasing and decreasing factors to speed up the algorithm.

\section{Desirable Properties of Market Mechanism}

It is necessary and important to evaluate the quality of a market-clearing mechanism by checking the four desirable properties, which are market efficiency ${ }^{1}$, revenue adequacy ${ }^{2}$, incentive compatibility ${ }^{3}$ and cost recovery ${ }^{4}$. Based on the Hurwicz theorem [33], no mechanism is capable of achieving all those properties at the same time.

1) Market efficiency: After convergence, the total local residuals and the objective value of SO (16a) fall below global stopping criteria (19), thus the objective of the local optimization problem at optimum is

$$
\left\{\mathbf{E}_{n}^{*}, \mathbf{R}_{n}^{*}\right\}=\underset{\left\{\mathbf{E}_{n}, \mathbf{R}_{n}\right\}}{\operatorname{argmin}} C_{n}^{e}\left(E_{n}\right)+\tilde{C}_{n}^{e}\left(\mathbf{E}_{n}\right)+C_{n}^{r}\left(R_{n}\right)+\tilde{C}_{n}^{r}\left(\mathbf{R}_{n}\right)
$$

which means $\left\{\mathbf{E}_{n}^{*}, \mathbf{R}_{n}^{*}\right\}$ is the optimal solution to minimize the individual cost for agents $n$. The summation of above objective functions for all agents is aligned with the objective of total cost minimization problem as

$$
\begin{aligned}
& \underset{\left.\mathbf{E}_{n \in \Omega}^{*}, \mathbf{R}_{n \in \Omega}^{*}\right\}=}{\underset{\left\{\mathbf{E}_{n \in \Omega}, \mathbf{R}_{n \in \Omega}\right\}}{\operatorname{argmin}}} \sum_{n \in \Omega}\left(C_{n}^{e}\left(E_{n}\right)+\tilde{C}_{n}^{e}\left(\mathbf{E}_{n}\right)+C_{n}^{r}\left(R_{n}\right)+\tilde{C}_{n}^{r}\left(\mathbf{R}_{n}\right)\right) \\
& \text { (2) }
\end{aligned}
$$

which implies the individual optimal solutions also cooperatively minimize the total cost. Thus, the optimality of the solution of our adopted decentralized mechanism is proved, which also means market efficiency is satisfied.

2) Incentive compatibility: A market agent may gain profit by not trustfully offering in terms of price or quantity, but we assume that agents are truthful in this work. Our market mechanism is designed based on the Locational Marginal Price (LMP), and the biggest limitation of LMP markets is that the incentive-compatibility cannot be satisfied. But, we can punish the cheating behavior in the real-time market. For example, if the renewable agents reveal less needed reserve in the dayahead market to gain more profit, it has a risk that paying more money to compensate for its deficiency in the real-time market since the real-time prices are usually higher. Besides, the market operator can also charge extra money for renewable generation deficiency.

3) Revenue adequacy: From (18), the prices between agents $n$ and $m$ are identical, i.e., $\lambda_{n m}=\lambda_{m n}$ and $\nu_{n m}=$ $\nu_{m n}$, the quantities between agents $n$ and $m$ are balanced, i.e., $E_{n m}+E_{m n}=0$ and $R_{n m}+R_{m n}=0$. Thus, the revenue

\footnotetext{
${ }^{1}$ Market efficiency is maximized when market outcomes align with the maximum social welfare.

${ }^{2}$ Revenue adequacy implies that there is no financial deficit in the market.

${ }^{3} \mathrm{~A}$ mechanism is called incentive compatible if every participant can maximize its objective just by acting according to its true preferences.

${ }^{4}$ Cost recovery implies that individual profit is non-negative.
} 
adequacy is also ensured because there is no financial deficit between each pair of agents.

4) Cost recovery: Since the cost and utility functions are convex, monotonically increasing, and passing through the origin, the agent can always set $E_{n}=E_{n m}=0$ and $R_{n}=R_{n m}=0$ to avoid a negative profit. Thus the cost recovery is satisfied.

Summing up the above, our market satisfies most of the desirable properties.

\section{Renewable Community-based P2P Joint Market}

In practice, the uncertainties may correlate, especially negative correlation, i.e., the deficit and excess between outputs can compensate for each other. Therefore, to make full use of the complementarity, it is beneficial to collect all uncertainty distributions to yield the joint distribution and determine the total required reserve, which is highly likely less than the summation of reserve determined by each agent individually. All renewable agents can collaborate as a community as shown in Fig. 4. The renewable agents inside the community provide their uncertainty distributions to the community manager, who help calculate the total required reserve, which will be distributed by all renewable agents since they cooperate together. But the fully decentralized mechanism is not broken, each renewable agent can still participate the market individually without sharing cost function and real power generation.

\section{A. Modeling Uncertainty and Determining Reserve}

First of all, we need to model the joint uncertainty distribution of the renewable community. We set the whole uncertainty $Y$ as a linear summation of the uncertainties of all renewable agents as follows:

$$
Y=\mathbf{1}^{T} \tilde{\mathbf{R}}
$$

where $\tilde{\mathbf{R}}$ denotes the random vector consisting of multiple uncertainty distributions. Inspired by [34], we adopt GMM to represent correlated non-Gaussian random variables. A GMM for $Y$ is defined as a convex combination of multi-dimensional Gaussian distribution functions with an adjustable parameter set $\Gamma=\left\{\varpi_{l}, \mu_{l}, \Sigma_{l} \mid l=1, \ldots, L\right\}$ :

$$
\begin{aligned}
& f_{Y}(y)=\sum_{l=1}^{L} \varpi_{l} N_{l}\left(y ; \mathbf{1}^{T} \mu_{l}, \mathbf{1}^{T} \Sigma_{l} \mathbf{1}\right), \quad \sum_{l=1}^{L} \varpi_{l}=1, \varpi>0 \\
& N_{l}\left(y ; \mathbf{1}^{T} \mu_{l}, \mathbf{1}^{T} \Sigma_{l} \mathbf{1}\right)=\frac{e^{-\frac{1}{2}\left(y-\mathbf{1}^{T} \mu_{l}\right)^{T}\left(\mathbf{1}^{T} \Sigma_{l} \mathbf{1}\right)^{-1}\left(y-\mathbf{1}^{T} \mu_{l}\right)}}{(2 \pi)^{W / 2} \operatorname{det}\left(\mathbf{1}^{T} \Sigma_{l} \mathbf{1}\right)^{1 / 2}}
\end{aligned}
$$

where $N_{l}(\cdot)$ denotes the multivariate Gaussian distribution function. Each Gaussian distribution function is called a Gaussian component. The $\mathrm{CDF}$ of $Y$ is given by

$$
F_{Y}(y)=\sum_{l=1}^{L} \varpi_{l}\left[\int_{v \leq y} N_{l}\left(y ; \mathbf{1}^{T} \mu_{l}, \mathbf{1}^{T} \Sigma_{l} \mathbf{1}\right) d v\right]
$$

Using historical data to estimate the parameter set $\Gamma$ of a GMM is a typical maximum likelihood estimation (MLE) problem, which can be solved by expectation maximization (EM) algorithm [35]. We use the Bayesian Information Criterion (BIC) [36] to decide the number of components in GMM, which is attained when the BIC reaches the minimal value.

Then, we will introduce how to find a closed form of CDF $F_{Y}(y)$. A piece-wise fourth-order polynomial approximation is adopted [34], as shown in (25), to approximate the CDF of a Gaussian random variable with mean $\mathbf{1}^{T} \mu_{j}$ and standard derivation $\left(\mathbf{1}^{T} \Sigma_{l} \mathbf{1}\right)^{1 / 2}$. For simplicity, the polynomial function is denoted as

$$
\int_{v \leq y} N_{l}\left(y ; \mathbf{1}^{T} \mu_{l}, \mathbf{1}^{T} \Sigma_{l} \mathbf{1}\right) d v \approx \operatorname{poly}_{l}\left(\frac{y-\mathbf{1}^{T} \mu_{l}}{\sqrt{\mathbf{1}^{T} \Sigma_{l} \mathbf{1}}}\right)
$$

Thus, we have

$$
F_{Y}(y)=\sum_{l=1}^{L} \varpi_{l} \operatorname{poly}_{l}\left(\frac{y-\mathbf{1}^{T} \mu_{l}}{\sqrt{\mathbf{1}^{T} \Sigma_{l} \mathbf{1}}}\right)=\operatorname{poly}_{\Sigma}(y)
$$

(30) indicates that the CDF of $Y$ is a convex combination of multiple fourth-order polynomials. This expression is analytical and can be computed quickly. Finding the inverse CDF of $Y$ is equal to finding a root of the quartic polynomial poly $y_{\Sigma}^{-1}(\cdot)$. From above, the total required reserve $\mathbb{R}$ is finally obtained as

$$
1-\operatorname{poly}_{\Sigma}(\mathbb{R}) \geq 1-\epsilon \Rightarrow \mathbb{R} \leq \operatorname{poly}_{\Sigma}^{-1}(\epsilon)
$$

and we can set $\mathbb{R}=\operatorname{poly}_{\Sigma}^{-1}(\epsilon)$ for this renewable community.

\section{B. Local Optimization Problem for Renewable Agent}

After obtaining the total required reserve $\mathbb{R}$, it will be distributed by all renewable agents according to the proportion of individual required reserve to the total required reserve before cooperating. Mathematically, the required reserve for agent $n$ is $\frac{R_{n}}{\sum_{n \in \Omega} R_{n}} \mathbb{R}$, where $R_{n}=\gamma-\frac{1}{\alpha} \ln \left(\epsilon_{n}^{-1 / \beta}-1\right), \mathbb{R}=$ poly $_{\Sigma}^{-1}(\epsilon)$. Since $\mathbb{R} \leq \sum_{n \in \Omega} R_{n}$, we have $\frac{R_{n}}{\sum_{n \in \Omega} R_{n}} \mathbb{R} \leq R_{n}$. Thus each agent can buy less reserve, they are willing to participate the community and share the uncertainty information. The local optimization problem for renewable agent $n$ at a given iteration $k$ is

$$
\left\{\mathbf{E}_{n}^{k+1}, \mathbf{R}_{n}^{k+1}\right\}=
$$

$$
\begin{gathered}
\int_{v \leq y} N_{l}\left(y ; \mu_{l}, \Sigma_{l}\right) d v \approx\left\{\begin{array}{cc}
0.5+\left(h_{4} \tilde{y}_{l}^{4}+h_{3} \tilde{y}_{l}^{3}+h_{2} \tilde{y}_{l}^{2}+h_{1} \tilde{y}_{l}+h_{0}\right), & 0<\tilde{y}_{l}<3 \\
0.5-\left(h_{4} \tilde{y}_{l}^{4}-h_{3} \tilde{y}_{l}^{3}+h_{2} \tilde{y}_{l}^{2}-h_{1} \tilde{y}_{l}+h_{0}\right), & -3<\tilde{y}_{l}<0 \\
1, & \tilde{y}_{l}>3 \\
0, & \tilde{y}_{l}<-3
\end{array}\right. \\
\tilde{y}_{l}=\left(y-\mu_{l}\right) / \sqrt{\Sigma_{l}}, h_{4}=0.00709, h_{3}=-0.02918, h_{2}=-0.06211, h_{1}=0.424, h_{0}=-0.0005
\end{gathered}
$$




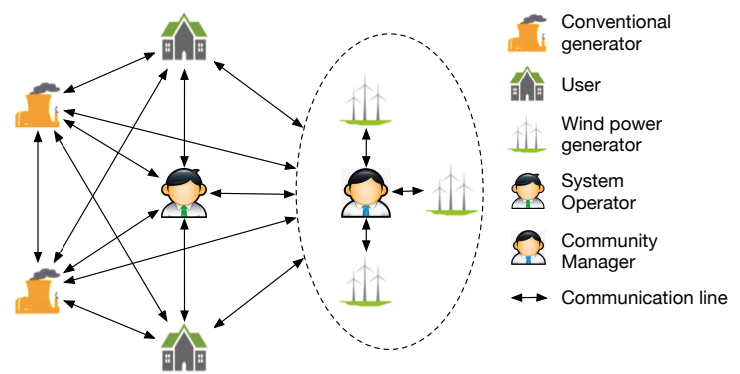

Fig. 4: Renewable community-based P2P joint market

$$
\begin{aligned}
& \underset{\left\{\mathbf{E}_{n}, \mathbf{R}_{n}\right\}}{\operatorname{argmin}} \tilde{C}_{n}^{e}\left(\mathbf{E}_{n}\right)+\tilde{C}_{n}^{r}\left(\mathbf{R}_{n}\right)+ \\
& \sum_{m \in \omega_{n}}\left[\lambda_{n m}^{k}\left(\frac{E_{n m}^{k}-E_{m n}^{k}}{2}-E_{n m}\right)+\nu_{n m}^{k}\left(\frac{R_{n m}^{k}-R_{m n}^{k}}{2}-R_{n m}\right)\right. \\
& \left.+\frac{\rho}{2}\left(\frac{E_{n m}^{k}-E_{m n}^{k}}{2}-E_{n m}\right)^{2}+\frac{\tau}{2}\left(\frac{R_{n m}^{k}-R_{m n}^{k}}{2}-R_{n m}\right)^{2}\right] \\
& +v_{i}^{k}\left(E_{n}+E_{-n}^{k}-\sum_{(i, j) \in \mathcal{L}} P_{i j}^{k+1}\right)+\frac{\phi}{2}\left(E_{n}+E_{-n}^{k}-\sum_{(i, j) \in \mathcal{L}} P_{i j}^{k+1}\right)^{2}
\end{aligned}
$$

$$
\begin{array}{ll}
\text { s.t. } & E_{n}^{f}=\sum_{m \in \omega_{n}} E_{n m} \\
& \frac{R_{n}}{\sum_{n \in \Omega} R_{n}} \mathbb{R}=\sum_{m \in \omega_{n}} R_{n m} \\
& E_{n m} \geq 0, R_{n m} \leq 0
\end{array}
$$

\section{Simulation Results}

As a basis for illustration and discussion, we first consider a small market to show the outcomes and comparison with other market mechanisms. Then real renewable output data is employed to show the convergence performance in a larger market. The improvement by employing adaptive penalty factor is also tested. Finally, the scalability is demonstrated by increasing the number of agents. For the aim of better formulation and simulation, we model the cost and utility as quadratic functions (33), though our mechanism is still applicable for any strictly convex functions.

$$
\left\{\begin{aligned}
C_{n}^{e}\left(E_{n}\right) & =\frac{1}{2} a_{n}^{e} E_{n}^{2}+b_{n}^{e} E_{n} \\
C_{n}^{r}\left(R_{n}\right) & =\frac{1}{2} a_{n}^{r} R_{n}^{2}+b_{n}^{r} R_{n}
\end{aligned}\right.
$$

To better show the performance, uniform distribution stochastic parameter settings are applied. We perform simulations using Matlab R2017b on a PC with $1.6 \mathrm{GHz}$ Intel Core 4 Duo CPU and 8 GB memory.

\section{A. Case Study}

For ease of illustration, we first consider a small market with 10 agents on the IEEE 9-bus test system as shown in Fig. 5. The parameters of agents are summarized in Table. I. The susceptance $Y$ and line capacity limit $C$ are setting to 3 and 10 for all lines. Agent G1-G3 are conventional generators, agent

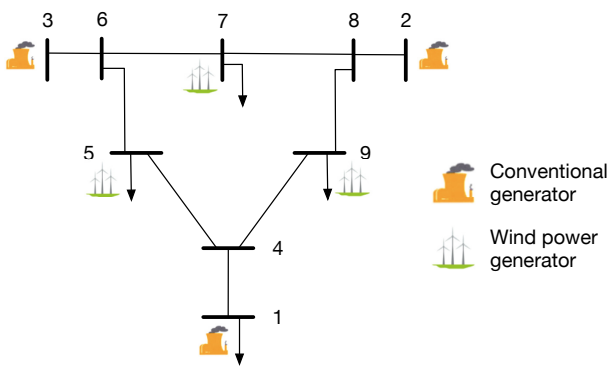

Fig. 5: IEEE 9-bus test system used for case study

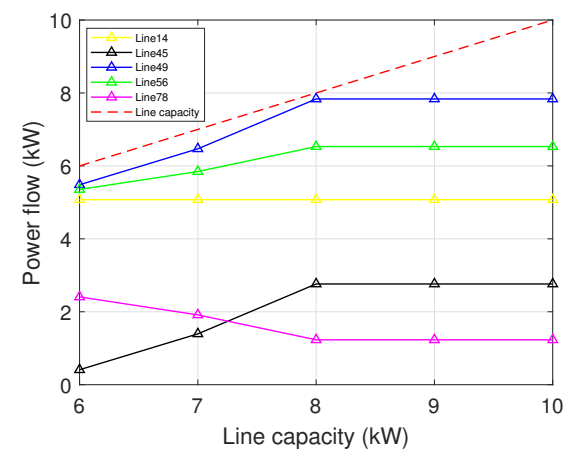

Fig. 6: Power flow in different lines for different line capacities

U1-U4 are users, and agent R1-R3 are wind power generator. The trading coefficient $c_{n m}$ varies from 0 to 0.3 . The penalty factors $\rho, \tau$ and $\phi$ are all set to 1 , the acceptable probability $\epsilon$ is 0.05 , and all global stopping criteria are chosen to be $10^{-3}$.

1) Final Outcomes: The final traded quantities and prices between agents are shown in Table. II. The revenue and payment are calculated by price multiplying quantity. The prices are different between agents, showing the product differentiation. It can be also seen that the energy demand of users are all satisfied by the wind power generators since renewable generation cost is much lower, and the reserve demand of renewable generators are all provided by conventional generators because the demand curtailment cost is higher.

2) Impact of the Line Capacity: Impact of the line capacity limit on the power flow is studied. In the considered test system, the power flows in lines connecting bus 1 and 4,4 and 5, 4 and 9,5 and 6,7 and 8, are non-zero, thus the results are only shown for these lines. The maximum line capacity for these lines ranges from $6-10 \mathrm{~kW}$. The results are illustrated in Fig. 6 and confirm that the power flow in these lines is always not larger than the maximum line capacity, which means the proposed algorithm can control line flow constraints in the $\mathrm{P} 2 \mathrm{P}$ market. In the case there is a congested line in the network, agents will avoid transactions that need to use the congested line since they have to pay an additional network charge.

3) Comparison with Other Markets: We make a comparison with the renewable community-based and pool-based markets. Seen from Table. III, the social costs of the P2P market and pool-based market are higher than that of the community-based market because the total required reserve is highly reduced (11.79 to 4.53 ), the cost for producing reserve decreases correspondingly. The social cost of pool-based 
TABLE I: Agents' characteristics of simple case study

\begin{tabular}{cccccccccc}
\hline \hline Agent & Bus & $a_{n}^{e}\left[\$ / \mathrm{kW}^{2}\right]$ & $b_{n}^{e}[\$ / \mathrm{kW}]$ & $a_{n}^{r}\left[\$ / \mathrm{kW}^{2}\right]$ & $b_{n}^{r}[\$ / \mathrm{kW}]$ & $\underline{E_{n}[\mathrm{~kW}]}$ & $\overline{E_{n}}[\mathrm{~kW}]$ & $\underline{R_{n}}[\mathrm{~kW}]$ & $\overline{R_{n}}[\mathrm{~kW}]$ \\
\hline \hline G1 & 1 & 0.0268 & 18.1712 & 0.0153 & 6.0845 & 0 & 26.6146 & 0 & 4.4811 \\
G2 & 2 & 0.0325 & 13.4665 & 0.0133 & 6.2985 & 0 & 27.9591 & 0 & 4.7943 \\
G3 & 3 & 0.0334 & 19.0183 & 0.0120 & 5.1931 & 0 & 22.4487 & 0 & 7.5596 \\
\hline $\mathrm{U} 1$ & 1 & 0.0300 & 12.7700 & 0.0153 & 7.8712 & -28.2561 & -5.0738 & 0 & 6.8111 \\
$\mathrm{U} 2$ & 5 & 0.0301 & 13.8127 & 0.0106 & 6.7929 & -24.6859 & -6.0175 & 0 & 7.2551 \\
$\mathrm{U} 3$ & 7 & 0.0279 & 10.5358 & 0.0138 & 8.8750 & -23.3060 & -9.5611 & 0 & 5.2768 \\
$\mathrm{U} 4$ & 9 & 0.0266 & 12.0424 & 0.0177 & 5.3499 & -39.0007 & -5.7910 & 0 & 5.1458 \\
\hline $\mathrm{R} 1$ & 5 & 0.0134 & 5.3529 & 0 & 1 & 15.1209 & 15.1209 & -3.6904 & -3.6904 \\
R2 & 7 & 0.0136 & 7.3221 & 0 & 1 & 17.3210 & 17.3210 & -4.3789 & -4.3789 \\
R3 & 9 & 0.0137 & 6.0183 & 0 & 1 & 12.3949 & 12.3949 & -3.7179 & -3.7179 \\
\hline \hline
\end{tabular}

TABLE II: Final dispatches in terms of energy and reserve

(a) Traded quantities and prices of energy

\begin{tabular}{lccc}
\hline & $\mathrm{R} 1$ & $\mathrm{R} 2$ & $\mathrm{R} 3$ \\
\hline \hline $\mathrm{U} 1$ & $/$ & $5.07 \mathrm{~kW} / 13.14 \$ / \mathrm{kW}$ & $/$ \\
$\mathrm{U} 2$ & $0.47 \mathrm{~kW} / 12.85 \$ / \mathrm{kW}$ & $11.56 \mathrm{~kW} / 12.98 \$ / \mathrm{kW}$ & $12.39 \mathrm{~kW} / 13.05 \$ / \mathrm{kW}$ \\
$\mathrm{U} 3$ & $/$ & $9.56 \mathrm{~kW} / 12.91 \$ / \mathrm{kW}$ & $/$ \\
$\mathrm{U} 4$ & $/$ & $5.79 \mathrm{~kW} / 13.13 \$ / \mathrm{kW}$ & $/$ \\
\hline \hline
\end{tabular}

(b) Traded quantities and prices of reserve

\begin{tabular}{cccc}
\hline \hline & $\mathrm{G} 1$ & $\mathrm{G} 2$ & $\mathrm{G} 3$ \\
\hline \hline $\mathrm{R} 1$ & $3.69 \mathrm{~kW} / 6.16 \$ / \mathrm{kW}$ & $/$ & $/$ \\
$\mathrm{R} 2$ & $0.54 \mathrm{~kW} / 6.22 \$ / \mathrm{kW}$ & $/$ & $3.84 \mathrm{~kW} / 6.19 \$ / \mathrm{kW}$ \\
$\mathrm{R} 3$ & $/$ & $/$ & $3.72 \mathrm{~kW} / 6.04 \$ / \mathrm{kW}$ \\
\hline \hline
\end{tabular}

market is lowest because the trading cost is not considered.

Then, we focus on the fairness of payment dispatches. The payments of energy clearing for three markets are all calculated by price multiplying quantity, which is fair enough. While for reserve, in the pool-based market, the reserve payments will be equally shared by all renewable agents [19], which is apparently not fair for agent whose uncertainty is less. Intuitively, the renewable agent should pay the bill in proportion to the uncertainty it brings in. To measure fairness, we adjust the payments according to the proportion of uncertainty, which is quantified by the reserve. The uncertainties are normalized by fixing the first one to 1 and denoted by $u_{n}$. An index based on Jain fairness index [37] is proposed to define the fairness of reserve payment dispatches:

$$
F=\frac{\left(\sum_{n \in \Omega_{r}} p_{n}^{r} / u_{n}\right)^{2}}{\left|\Omega_{r}\right| \sum_{n \in \Omega_{r}}\left(p_{n}^{r} / u_{n}\right)^{2}}
$$

where $p_{n}^{r}$ is the reserve payment of agent $n,\left|\Omega_{r}\right|$ is the number of renewable agents. The result ranges from $\frac{1}{\left|\Omega_{r}\right|}$ (worst case) to 1 (best case). Seen from the results, the full P2P and community-based markets are fair in reserve payment dispatches, while the fairness is 0.994 for the pool-based market, showing some unfairness.

\section{B. Application to Real Data}

We build a much larger market composed of 15 conventional generators, 15 users and 15 wind power generators with the
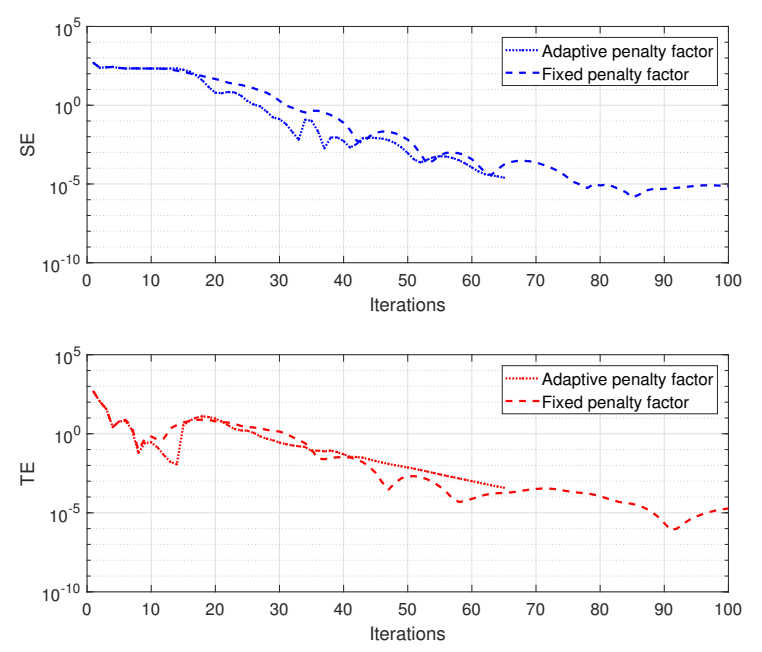

Fig. 7: Primal and dual energy residuals convergence process

real wind power scenarios data from [38]. We take 100 scenarios for each wind power generator to yield the uncertainty distribution. The forecast output is the mean value of the 100 scenarios and the installed capacity is set to $20 \mathrm{~kW}$.

Fig. 7-8 show the convergence performance, which is measured by the global residuals defined in (19)-(20). As long as they reach the stopping criteria, the balances of energy and reserve are satisfied, and the results remain unchanged between two iterations. It takes about 100 iterations to converge for fixed penalty factors. Some oscillations can be seen in the curves, this is because the changing tendencies of residuals between primal (energy) and dual (reserve) are in opposition to each other, i.e., the increase of one may cause a decrease of the other one. Therefore, we also depict the convergence processes of total residuals $(\mathrm{SE}+\mathrm{SR}+\mathrm{TE}+\mathrm{TR})$ in Fig. 9. It is shown that the total residuals continue dropping without oscillation. The acceleration performance of adaptive penalty factor for reducing communication burden is also tested. As shown in Fig. 9, the iterations are highly reduced from 102 to 65 by employing adaptive penalty factors.

\section{Scalability}

In practice, the $\mathrm{P} 2 \mathrm{P}$ market will be deployed for a large number of agents and the number of transactions would be 
TABLE III: Comparison results of three markets

\begin{tabular}{cccc}
\hline \hline Market mechanism & Full P2P & Renewable Community-based & Pool-based \\
\hline \hline Total costs[\$] & -210.12 & -242.70 & -220.68 \\
Total traded energy[kW.h] & 44.84 & 44.84 & 44.84 \\
Total traded reserve[kW.h] & 11.79 & 4.53 & 11.79 \\
Reserve payments[\$] & $23.100 / 27.269 / 22.877$ & $8.782 / 10.426 / 8.608$ & 24.170 \\
Normalized uncertainties & $1 / 1.187 / 1.005$ & $1 / 1.187 / 1.005$ & $1 / 1.187 / 1.005$ \\
Fairness for reserve payment & 1 & 1 & 0.994 \\
\hline \hline
\end{tabular}


Fig. 8: Primal and dual reserve residuals convergence process

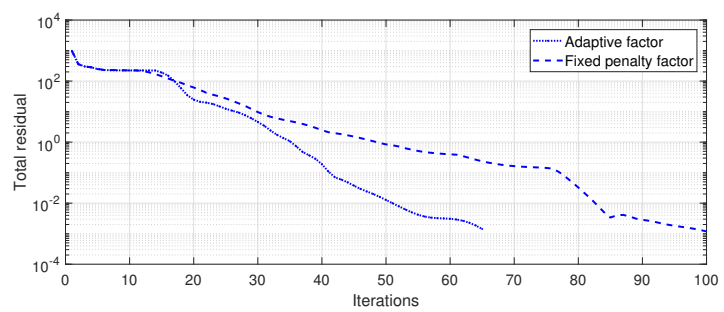

Fig. 9: Total residuals convergence process

significant. Therefore, the computation time of the algorithm and number of iterations are two critical factors affecting the scalability of the algorithm. To investigate the scalability issue, the proposed method is tested with higher number of agents. Fig. 10 shows the impact of number of agents, which ranges from 60 to 480, on the computational time of the proposed algorithm and number of iterations. Seen from the Fig. 10, both of the number of iterations and computational time increase with the number of agents. Since the scheduling of day-ahead market is for next day, the performance of scalability is acceptable and verifies our proposed mechanism is implementable for a market with large number of players. According to the fitting curve, the number of iterations and convergence time almost linearly increase with the number of agents, we can approximately estimate the time for convergence is still short enough even there are more than thousands of agents in the market.



(a)



(b)
Fig. 10: Impact of number of agents on iterations and time for convergence

\section{Vi. Conclusion, Limitation ANd Future Perspective}

\section{A. Conclusions}

P2P markets are considered as an evolution of the electricity markets driven by distributed energy resources and demand response management development. How to design a proper P2P market to take in the renewable resources with large uncertainty remains as a challenge. To this end, in this paper, a P2P joint energy and reserve market is proposed. Uncertainty is first modeled based on a versatile distribution, and reserve is determined based on chance-constrained optimization and negotiation. Then, a fully decentralized negotiation mechanism is proposed for market-clearing based on consensus ADMM. Furthermore, a renewable community-based P2P joint market is designed for utilizing the correlation and complementarity between uncertainties to further reduce social costs. In simulations, a simple case is tested to show that our market is economical, fair and privacy-friendly. Then a larger market with more agents and real wind power data is built, showing good convergence performance. Finally, the scalability of our market is verified by increasing the number of agents.

\section{B. Limitation and Future Perspective}

The main limitation of our methodology is that we consider the power flow and network constraints for the energy, but the feasible operating under any usage of reserve is not guaranteed. One workable approach is to reschedule the power flows in real-time market but requires high computational efficiency. Therefore, for future work, we will concentrate on improving the computational efficiency of $\mathrm{P} 2 \mathrm{P}$ mechanism so that we can design a real-time P2P market and schedule the power flows fast in response to uncertainty. 


\section{APPENDIX A}

\section{Decentralized Negotiation Mechanism}

The social cost minimization problem (11) can be rewritten in this form:

$$
\begin{array}{ll}
\min & \sum_{n \in \omega}\left(C_{n}^{e}\left(E_{n}\right)+\tilde{C}_{n}^{e}\left(\mathbf{E}_{n}\right)+C_{n}^{r}\left(R_{n}\right)+\tilde{C}_{n}^{r}\left(\mathbf{R}_{n}\right)\right) \\
\text { s.t. } & E_{n m}=-E_{m n} \quad \forall(n, m) \in\left(\Omega, \omega_{n}\right) \\
& R_{n m}=-R_{m n} \quad \forall(n, m) \in\left(\Omega, \omega_{n}\right) \\
& \sum_{n \in \mathcal{N}_{i}} E_{n}=\sum_{(i, j) \in \mathcal{L}} P_{i j}^{k+1} \quad \forall i \in \mathcal{N}
\end{array}
$$

For the constraints (35b)-(35c), each pair of agents should reach a consensus on the trading of energy and reserve. According to the consensus ADMM algorithm in [32], the update process is written as

$$
\begin{aligned}
x^{k+1} & :=\underset{x}{\operatorname{argmin}}\left(f(x)+y^{k}\left(x-\bar{x}^{k}\right)+(\rho / 2)\left\|x-\bar{x}^{k}\right\|_{2}^{2}\right) \\
y^{k+1} & :=y^{k}+\rho\left(x^{k+1}-\bar{x}^{k+1}\right)
\end{aligned}
$$

We set $x=\left\{E_{n m}, R_{n m}\right\}, \bar{x}^{k}=\left\{\frac{E_{n m}^{k}-E_{m n}^{k}}{2}, \frac{R_{n m}^{k}-R_{m n}^{k}}{2}\right\}$ and $y=\left\{\lambda_{n m}, \tau_{n m}\right\}$. After simplifications, the decentralized negotiation between agents for the energy and reserve reads as the former part of local optimization problem (17a). For the constraint (35c), the agents at same bus $i$ should cooperatively satisfy the power balance. Using standard ADMM structure, the decentralized mechanism reads as the latter part of local optimization problem (17b).

\section{REFERENCES}

[1] J. Hu, R. Harmsen, W. Crijns-Graus, E. Worrell, and M. van den Broek, "Identifying barriers to large-scale integration of variable renewable electricity into the electricity market: A literature review of market design," Renewable and Sustainable Energy Reviews, vol. 81, pp. 2181$2195,2018$.

[2] J. Kang, R. Yu, X. Huang, S. Maharjan, Y. Zhang, and E. Hossain, "Enabling localized peer-to-peer electricity trading among plug-in hybrid electric vehicles using consortium blockchains," IEEE Transactions on Industrial Informatics, vol. 13, no. 6, pp. 3154-3164, 2017.

[3] S. Wang, A. F. Taha, J. Wang, K. Kvaternik, and A. Hahn, "Energy crowdsourcing and peer-to-peer energy trading in blockchain-enabled smart grids," IEEE Transactions on Systems, Man, and Cybernetics: Systems, vol. 49, no. 8, pp. 1612-1623, 2019.

[4] T. Morstyn and M. D. McCulloch, "Multiclass energy management for peer-to-peer energy trading driven by prosumer preferences," IEEE Transactions on Power Systems, vol. 34, no. 5, pp. 4005-4014, 2018.

[5] R. Alvaro-Hermana, J. Fraile-Ardanuy, P. J. Zufiria, L. Knapen, and D. Janssens, "Peer to peer energy trading with electric vehicles," IEEE Intelligent Transportation Systems Magazine, vol. 8, no. 3, pp. 33-44, 2016.

[6] C. Zhang, J. Wu, Y. Zhou, M. Cheng, and C. Long, "Peer-to-peer energy trading in a microgrid," Applied Energy, vol. 220, pp. 1-12, 2018.

[7] A. Paudel, K. Chaudhari, C. Long, and H. B. Gooi, "Peer-to-peer energy trading in a prosumer-based community microgrid: A game-theoretic model," IEEE Transactions on Industrial Electronics, vol. 66, no. 8, pp. 6087-6097, 2018.

[8] T. Morstyn, A. Teytelboym, and M. D. McCulloch, "Bilateral contract networks for peer-to-peer energy trading," IEEE Transactions on Smart Grid, vol. 10, no. 2, pp. 2026-2035, 2018.

[9] T. Baroche, P. Pinson, R. L. G. Latimier, and H. B. Ahmed, "Exogenous cost allocation in peer-to-peer electricity markets," IEEE Transactions on Power Systems, vol. 34, no. 4, pp. 2553-2564, 2019.

[10] M. Khorasany, Y. Mishra, and G. Ledwich, "A decentralized bilateral energy trading system for peer-to-peer electricity markets," IEEE Transactions on Industrial Electronics, vol. 67, no. 6, pp. 4646-4657, 2020.
[11] T. Sousa, T. Soares, P. Pinson, F. Moret, T. Baroche, and E. Sorin, "Peer-to-peer and community-based markets: A comprehensive review," Renewable and Sustainable Energy Reviews, vol. 104, pp. 367-378, 2019.

[12] F. Moret, T. Baroche, E. Sorin, and P. Pinson, "Negotiation algorithms for peer-to-peer electricity markets: computational properties," in 2018 Power Systems Computation Conference (PSCC), pp. 1-7, 2018.

[13] R. L. G. Latimier, T. Baroche, and H. B. Ahmed, "Mitigation of communication costs in peer-to-peer electricity markets," in 2019 IEEE Milan PowerTech, pp. 1-6, 2019.

[14] F. Moret and P. Pinson, "Energy collectives: a community and fairness based approach to future electricity markets," IEEE Transactions on Power Systems, vol. 34, no. 5, pp. 3994-4004, 2019.

[15] E. Sorin, L. Bobo, and P. Pinson, "Consensus-based approach to peer-topeer electricity markets with product differentiation," IEEE Transactions on Power Systems, vol. 34, no. 2, pp. 994-1004, 2018.

[16] M. Caramanis, E. Ntakou, W. W. Hogan, A. Chakrabortty, and J. Schoene, "Co-optimization of power and reserves in dynamic T\&D power markets with nondispatchable renewable generation and distributed energy resources," Proceedings of the IEEE, vol. 104, no. 4, pp. 807-836, 2016.

[17] N. G. Cobos, J. M. Arroyo, N. Alguacil, and J. Wang, "Robust energy and reserve scheduling considering bulk energy storage units and wind uncertainty," IEEE Transactions on Power Systems, vol. 33, no. 5, pp. 5206-5216, 2018.

[18] S. S. Reddy, P. R. Bijwe, and A. R. Abhyankar, "Joint energy and spinning reserve market clearing incorporating wind power and load forecast uncertainties," IEEE Systems Journal, vol. 9, no. 1, pp. 152$164,2015$.

[19] F. Bona, N. Gast, J.-Y. Le Boudec, P. Pinson, and D.-C. Tomozei, "Attribution mechanisms for ancillary service costs induced by variability in power delivery," IEEE Transactions on Power Systems, vol. 32, no. 3, pp. 1891-1901, 2016.

[20] K. Zhang, S. Troitzsch, S. Hanif, and T. Hamacher, "Coordinated market design for peer-to-peer energy trade and ancillary services in distribution grids," IEEE Transactions on Smart Grid, pp. 1-1, 2020.

[21] S. Bjarghov, C. Ø. Naversen, K. Thorvaldsen, and H. Farahmand, "A three-stage stochastic peer-to-peer market clearing model with real-time reserve activation," arXiv preprint arXiv:1910.10951, 2019.

[22] Z. S. Zhang, Y. Z. Sun, D. W. Gao, J. Lin, and L. Cheng, "A versatile probability distribution model for wind power forecast errors and its application in economic dispatch," IEEE Transactions on Power Systems, vol. 28 , no. 3, pp. 3114-3125, 2013.

[23] R. H. Day, "Rational choice and economic behavior," Theory and Decision, vol. 1, no. 3, pp. 229-251, 1971.

[24] T. Soares, P. Pinson, T. V. Jensen, and H. Morais, "Optimal offering strategies for wind power in energy and primary reserve markets," IEEE Transactions on Sustainable Energy, vol. 7, no. 3, pp. 1036-1045, 2016.

[25] S. S. Reddy, P. Bijwe, and A. R. Abhyankar, "Joint energy and spinning reserve market clearing incorporating wind power and load forecast uncertainties," IEEE Systems Journal, vol. 9, no. 1, pp. 152-164, 2013.

[26] N. Gatsis and G. B. Giannakis, "Decomposition Algorithms for Market Clearing With Large-Scale Demand Response," IEEE Transactions on Smart Grid, vol. 4, no. 4, pp. 1976-1987, 2013.

[27] A. Kargarian, J. Mohammadi, J. Guo, S. Chakrabarti, M. Barati, G. Hug, S. Kar, and R. Baldick, "Toward Distributed/Decentralized DC Optimal Power Flow Implementation in Future Electric Power Systems," IEEE Transactions on Smart Grid, vol. 9, no. 4, pp. 2574-2594, 2018.

[28] F. Bouffard and F. D. Galiana, "Stochastic security for operations planning with significant wind power generation," IEEE Transactions on Power Systems, vol. 23, no. 2, pp. 306-316, 2008.

[29] J. Zhang, B.-M. Hodge, and A. Florita, "Joint probability distribution and correlation analysis of wind and solar power forecast errors in the western interconnection," Journal of Energy Engineering, vol. 141, no. 1 , 2015.

[30] D. Thevenard and S. Pelland, "Estimating the uncertainty in long-term photovoltaic yield predictions," Solar energy, vol. 91, pp. 432-445, 2013.

[31] L. Liu, Y. Zhao, D. Chang, J. Xie, Z. Ma, Q. Sun, H. Yin, and R. Wennersten, "Prediction of short-term pv power output and uncertainty analysis," Applied energy, vol. 228, pp. 700-711, 2018.

[32] S. Boyd, N. Parikh, E. Chu, B. Peleato, and J. Eckstein, "Distributed optimization and statistical learning via the alternating direction method of multipliers," Foundations and Trends in Machine Learning, vol. 3, no. 1, pp. 1-122, 2010.

[33] L. Hurwicz, "On informationally decentralized systems," Decision and organization: A volume in Honor of J. Marschak, 1972. 
[34] Z. Wang, C. Shen, F. Liu, X. Wu, C. C. Liu, and F. Gao, "Chanceconstrained economic dispatch with non-Gaussian correlated wind power uncertainty," IEEE Transactions on Power Systems, vol. 32, no. 6, pp. 4880-4893, 2017.

[35] R. Singh, B. C. Pal, and R. A. Jabr, "Loads using gaussian mixture model," IEEE Transactions on Power Systems, vol. 25, no. 1, pp. 2937, 2010.

[36] C. Keribin, "Consistent estimation of the order of mixture models," Sankhyā: The Indian Journal of Statistics, Series A, pp. 49-66, 2000.

[37] M. Dianati, X. Shen, and S. Naik, "A new fairness index for radio resource allocation in wireless networks," in IEEE Wireless Communications and Networking Conference, 2005, vol. 2, pp. 712-717, 2005.

[38] P. Pinson, "Wind energy: Forecasting challenges for its operational management," Statistical Science, vol. 28, no. 4, pp. 564-585, 2013.

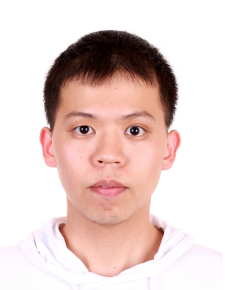

Zhenwei Guo received the B.S. degree in automation from Zhejiang University, Hangzhou, China, in 2016. Currently, he is pursuing the Ph.D. degree in the College of Control Science and Engineering, at Zhejiang University. He is a member of the Group of Networked Sensing and Control in the State Key Laboratory of Industrial Control Technology at Zhejiang University. His research interests include demand side management, distributed optimization and electricity market in smart grid.



Pierre Pinson (SM'13, F'20) received the M.Sc. degree in applied mathematics from the National Institute for Applied Sciences, Toulouse, France, and the $\mathrm{Ph} . \mathrm{D}$. degree in energetics from Ecole des Mines de Paris, Paris, France. He is a Professor with the Centre for Electric Power and Energy, Department of Electrical Engineering, Technical University of Denmark, Lyngby, Denmark, also heading a group focusing on energy analytics and markets. His research interests include forecasting, uncertainty estimation, optimization under uncertainty, decision sciences, and renewable energies. He is the Editor-in-Chief for the International Journal of Forecasting.

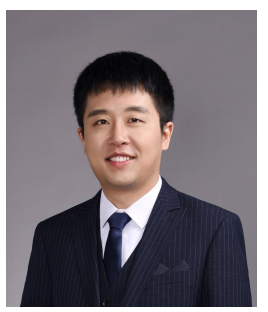

Shibo Chen (M'17) received the B.Eng. degree in electronic engineering from University of Science and Technology of China (USTC), Hefei, China, in 2011 and the Ph.D. degree in electronic and computer engineering from the Hong Kong University of Science and Technology (HKUST), Kowloon, Hong Kong, in 2017. He was a Postdoctoral Fellow with HKUST before joining the Department of Mechanical and Energy Engineering, Southern University of Science and Technology (SUSTech), Shenzhen, China in 2019 as a Research Assistant Professor. His current research interests include smart grid, optimization theory and game theory.

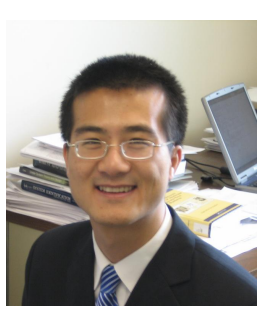

Qinmin Yang received the Bachelor's degree in Electrical Engineering from Civil Aviation University of China, Tianjin, China in 2001, the Master of Science Degree in Control Science and Engineering from Institute of Automation, Chinese Academy of Sciences, Beijing, China in 2004, and the Ph.D. degree in Electrical Engineering from the University of Missouri-Rolla, MO USA, in 2007. From 2007 to 2008, he was a Post-doctoral Research Associate at University of Missouri-Rolla. From 2008 to 2009, he was a system engineer with Caterpillar Inc. From 2009 to 2010, he was a Post-doctoral Research Associate at University of Connecticut. Since 2010, he has been with the State Key Laboratory of Industrial Control Technology, the College of Control Science and Engineering, Zhejiang University, China, where he is currently a professor. He has also held visiting positions in University of Toronto and Lehigh University. He has been serving as an Associate Editor for IEEE Transactions on Systems, Man, and Cybernetics: Systems, Transactions of the Institute of Measurement and Control, and Automatica Sinica. His research interests include intelligent

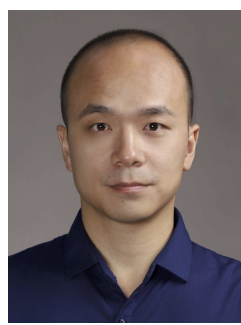

Zaiyue Yang (M'10) received the B.S. and M.S. degrees from the Department of Automation, University of Science and Technology of China, Hefei, China, in 2001 and 2004, respectively, and the Ph.D. degree from the Department of Mechanical Engineering, University of Hong Kong, in 2008. He was a Postdoctoral Fellow and Research Associate with the Department of Applied Mathematics, Hong Kong Polytechnic University, before joining the College of Control Science and Engineering, Zhejiang University, Hangzhou, China, in 2010. Then, he joined the Department of Mechanical and Energy Engineering, Southern University of Science and Technology, Shenzhen, China, in 2017. He is currently a Professor there. His current research interests include smart grid, signal processing and control theory. Prof. Yang is an associate editor for the IEEE Transactions on Industrial Informatics. 